\title{
Research on the Solution of Cell Invasion Model with Free Boundary
}

\author{
Rouzimaimaiti Mahemuti ${ }^{1}$, Ahmadjan Muhammadhajii ${ }^{*}$, Takashi Suzuki² \\ ${ }^{1}$ College of Mathematics and System Sciences, Xinjiang University, Urumqi, China \\ ${ }^{2}$ Graduate School of Engineering Science, Osaka University, Osaka, Japan \\ Email: ruyghar@outlook.com, ^ahmadjanm@gmail.com
}

How to cite this paper: Mahemuti, R., Muhammadhaji, A. and Suzuki, T. (2017) Research on the Solution of Cell Invasion Model with Free Boundary. Open Journal of Applied Sciences, 7, 242-261. https://doi.org/10.4236/ojapps.2017.76021

Received: April 14, 2017

Accepted: June 16, 2017

Published: June 19, 2017

Copyright () 2017 by authors and Scientific Research Publishing Inc. This work is licensed under the Creative Commons Attribution International License (CC BY 4.0).

http://creativecommons.org/licenses/by/4.0/

(c) (i) Open Access

\begin{abstract}
There are many works (i.e. [1]) aiming to find out numerically how positive feedback affects the formation of invadopodia and invasion of cancer cells; however, studies on the cancer cell invasion model with free boundary are fairly rare. In this paper, we study modified cancer cell invasion model with free boundary, where, free boundary stands for cancer cell membrane, so that we can more precisely describe the positive feedback affects. Firstly, we simplized the model by means of characteristic curve and semi-groups' property, and obtained the Stefan-like problem by introducing Gaussian Kernel and Green function. Secondly, based on the classical Stefan problem, we derived the integral solution of simplified model, which could lead us a further step to find the solution of modified cancer cell invasion model.
\end{abstract}

\section{Keywords}

Invadopodia, Cancer, Stefan Problem, Free Boundary

\section{Introduction}

As well known, cancer disease is one of the leading causes of death worldwide. Many natural and man-made factors (for example, smoking, car exhaust fumes, ultraviolet rays, air pollution and radiations, etc.) are the main risks for cancer disease. Metastasis is a leading death process, in which two processes are crucial from the viewpoint of cancer therapy. The first one is angiogenesis, nucleation of new blood vessels, which can provide enough nutrients to further development of tumor cells. The other one is tissue invasion. After the vascular growth of the tumor, cells become more aggressive that it can invade into the extracellular matrix, even into blood vessels, to complete the metastasis. Tissue invasion is a process in which cells can migrate and establish a new colony in new organs.

There are many studies about angiogenesis inhibition, because cancer cells 
have a certain size and cannot grow further without nutrients from blood vessels. Signaling molecule VEGF (vascular endothelial growth factor) can be secreted by cancer cells and can bind the normal endothelial cells to form new blood vessels. Scientists found inhibitors, such as bevacizumab, to block VEGF [2]. Bevacizumab binds to and disables VEGF to activate endothelial cells to create new blood vessels. This therapy is already applied clinically.

To reduce the ability of invasion is also one way to prevent metastasis. Cancer cells can spread by degrading ECMs. ECMs are degraded by the assembly of the actin cytoskeleton in invadopodia-the invasive feet of cancer cells. In fact, MMPs (matrix metalloproteinase), the family of ECM degrading enzymes [3], are up-regulated by signals from growth factors [4]. Then, after up-regulation of MMPs, actin assembly delivers it to the invasive site of cancer cells [5]. At the invasive site MT1-MMP (membrane-type MMP), part of MMPs, are responsible to cut laminin-5 [6]. 2 chains of laminin-5 after cleavage can bind to receptor molecules and send signals to drive actin assembly and MMP up-regulation.

Mathematical medicine and biology have become one of the popular topics in the study of modern applied mathematics. Where, cancer cell invasion models have received much attention in recent years [1] [7] [8] [9] [10] [11]. Research [7] has introduced a PDE model to observe the tissue invasion and tumor growth. Then research [8], based on the discrete-continuum hybrid simulation, promoted the study in [7] by considering the interactions, cell-cell adhesion and other essential functions of cells. There are many other studies relating to the growth of the tumor. For example, in [1], the authors are aiming to find out numerically how positive feedback affects the formation of invadopodia and invasion of cancer cells. They considered a model for the formation of invadopodia and reaction between proteins, such as act in monomers, ECM (extracellular matrix), signals and MMP (matrix metalloproteinases), which are playing significant role in cancer cell invasion. In the numerical simulation, the authors examined the effects of the molecules by varying the rate constants, and successfully reproduced invadopodia-like small protrusions, which have a similar scale of the real phenomenon, eventually, investigated the leading source of invadopodia; however, they did not study a boundary for cell body, which leads actins diffused into extracellular area.

To the best of our knowledge, studies on the cancer cell invasion model with free boundary are fairly rare. For that reasons, in this paper, we study a modified cancer cell invasion model with free boundary problem. The method used in this paper is motivated by Stefan problem.

In order to obtain cancer cell invasion model with free moving boundary, we need to consider the biological background of the problem. For the reader's convenience, we will introduce the process of invadopodia formation. Invadopodia are the invasive feet (as shown in Figure 1) of cancer cells which can degrade the surrounding matrix (mainly ECMs), and cause metastasis [12]. Invadopodia are enriched in act in filaments, which are cytoskeletal structures and pushing cell membrane to drive invadopodia. 


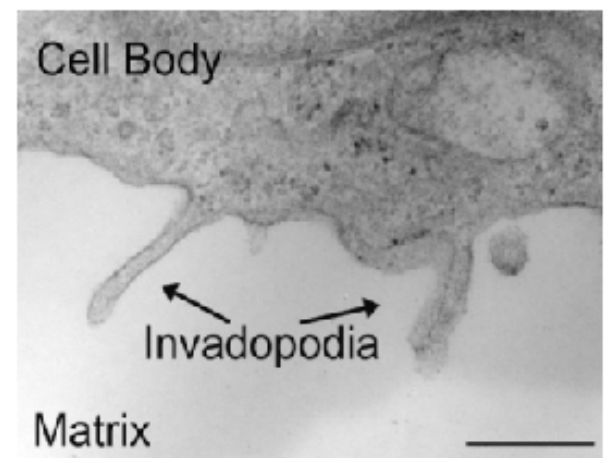

Figure 1. Invadopodia formation [8].

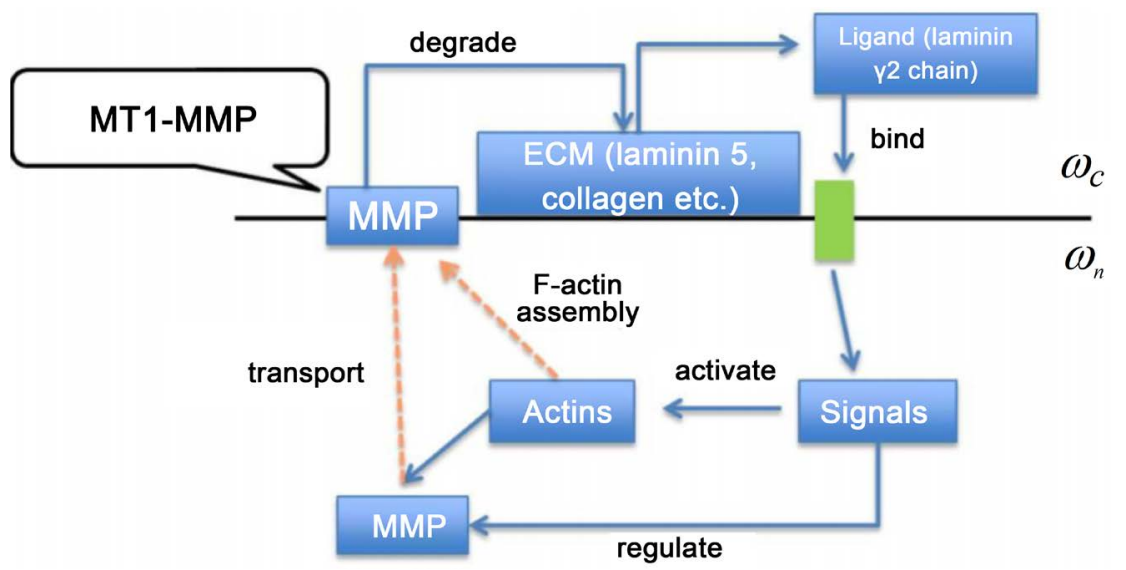

Figure 2. Cleavage of laminin 5 by MT1-MMP at juxtamembrane produce laminin- $\gamma 2$ which can bind to the receptor and can send signals.

ECM fragments are decreased by the reorganization of actin cytoskeleton indirectly [13]. Actins can transport MMPs to the invasive site of cancer cells, top of the invadopodia. MMPs can break and degrade the ECMs, broken ECM fragments in return bind with receptors on the cell membrane, and induce signals to reorganize actins and MMPs. Again, MMPs can break more ECMs, and become a positive feedback loop. We summarize the interactions as shown in Figure 2.

We improved the model considered in [1] by introduce a new variable $\sigma$ which stands for the signal droved by the connection of ECM fragments and receptors. ECM fragments are created near the membrane by degradation of ECMs. Hence, the corresponding mathematical model is described as follows:

$$
\begin{aligned}
& c_{t}+v \cdot \nabla c=-\kappa_{c} f c \\
& c_{t}^{*}+v \cdot \nabla c^{*}=\kappa_{c} f c
\end{aligned} \quad \text { on } \Gamma_{t},
$$

where, $\Gamma_{t}$ stands for the free moving boundary, $v$ stands for the boundary velocity, $c=c(x, t), c^{*}=c^{*}(x, t)$ and $f$ are stand for the concentration of ECM, ECM fragments and MMPs, respectively. ECM fragments (laminin $\gamma_{2}$ chains) bind to cell membrane receptors and send signals to the actin reorganization, where signals have random motility. Hence, we have

$$
\begin{aligned}
& \sigma_{t}=d_{\sigma} \Delta \sigma-\lambda_{\sigma} \sigma \\
& \left.\sigma\right|_{\Gamma_{t}}=c^{*}
\end{aligned}
$$


where, $\sigma=\sigma(x, t)$ represents signal concentration. The first equation of (2) describes the random motility and self decation of signals. The second equation of (2) describes ECM fragments bind to cell membrane and derive signals.

Free boundary $\Gamma_{t}$ is defined as

$$
\Gamma_{t}=\{x \in \Omega \mid \psi(x, t)=0\},
$$

where, $\psi$ is the level set function, and demands level set equation

$$
\psi_{t}+v \cdot \nabla \psi=0 .
$$

Since the membrane pushed by the F-actin which is reorganized by signals from cell receptors, therefore, the velocity of the membrane depends on the gradient of signals which cause F-actin polymerization. Hence, boundary velocity defined as follows:

$$
\nu=\gamma_{n} \nabla \sigma .
$$

Finally, we derived the modified cancer cell invasion model with cell boundary described as (1-5), our main purpose is to generalize this model into Stefan problem, then analytically discuss its solution.

The organization of this paper is as follows. In section 2 we present some basic definitions, assumptions and related properties, such as characteristic curve of the problem, Greens functions etc., to simplify the problem. In section 3, the main results, related theorem and its proof, of our paper was stated. Finally, the detailed calculation from (48) to (49) and (50) is given in Appendix A, B, C.

\section{Preliminaries}

The previous section, we stated the biological background and modified cancer cell invasion model with free moving boundary $(1-5)$. In this section, we will introduce some basic definitions and related preliminaries, such as Gaussian Kernel, Green function and derivation of Stefan problem etc., which would be useful in proving main results and solutions of Stefan problem (27) in section 3.

\subsection{Characteristic Curve}

In this paper, for simplicity, we take $f \equiv 1$, and discuss the model in 1 dimension. Hence, the level set Equation (4) is rewritten in the following form

$$
\frac{\mathrm{d}}{\mathrm{d} t} x_{ \pm}(t)+v\left(x_{ \pm}(t), t\right)=0,
$$

where, $x_{ \pm}(t)$ stand for the right and left side boundary positions, which depends on time $t, v_{ \pm}=v\left(x_{ \pm}(t), t\right)$ stand for the velocity of right and left side boundaries (Figure 3 ).

Take $y=U(t, s) x$, which satisfies

$$
\frac{\mathrm{d} y}{\mathrm{~d} t}=v(y, t),\left.\quad y\right|_{t=s}=x
$$

where $U(t, s)$ satisfies semi-group property:

$$
U(t, \tau) U(\tau, s)=U(t, s) .
$$




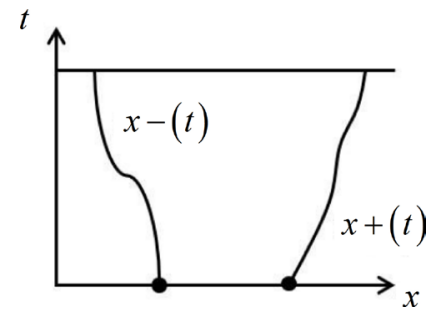

Figure 3. The positions of boundaries at time $t$.

Consider the following equation of $\mathcal{c}$,

$$
c_{t}+v \cdot c_{x}=-c \text { on } x_{ \pm}(t) .
$$

One can write

$$
\frac{\mathrm{d}}{\mathrm{d} t} c(y(t), t)=-c(y(t), t) .
$$

Then we easily have the solution

$$
c(y(t), t)=C \mathrm{e}^{-t}
$$

where $C$ is arbitrary constant. We take $t=s$, then we have

$$
C=c(y(s), s) \mathrm{e}^{s} .
$$

By (8), it follows that

$$
c(y(t), t)=c(y(s), s) \mathrm{e}^{s-t} .
$$

By $y=U(t, s) x$ and (9), we have

$$
c(U(t, s) x, t)=c(U(s, s) x, s) \mathrm{e}^{s-t} .
$$

When $s=0$,

$$
c(U(t, 0) x, t)=c_{0}(x) \mathrm{e}^{-t} .
$$

Now we take

$$
U(t, 0) x=z \Leftrightarrow U(0, t) z=x,
$$

and finally we can get

$$
c\left(x_{ \pm}(t), t\right)=c_{0}\left(U(0, t) x_{ \pm}(t)\right) \mathrm{e}^{-t} .
$$

By using an argument similar to the above, from the equation

$$
c_{t}^{*}+v \cdot c_{x}^{*}=c \quad \text { on } x_{ \pm}(t)
$$

we have the solution

$$
c^{*}\left(x_{ \pm}(t), t\right)=\left(1-\mathrm{e}^{-t}\right) c_{0}\left(U(0, t) x_{ \pm}(t)\right)+c_{0}^{*}\left(U(0, t) x_{ \pm}(t)\right) .
$$

Then signals $\sigma$ on boundary satisfies

$$
\sigma\left(x_{ \pm}(t), t\right)=c^{*}\left(x_{ \pm}(t), t\right)=\left(1-\mathrm{e}^{-t}\right) c_{0}\left(U(0, t) x_{ \pm}(t)\right)+c_{0}^{*}\left(U(0, t) x_{ \pm}(t)\right) .
$$

Finally we have,

$$
\sigma_{t}=\sigma_{x x}-\sigma, \text { in }\left(x_{-}(t), x_{+}(t)\right)
$$


with

$$
\sigma\left(x_{ \pm}(t), t\right)=\left(1-\mathrm{e}^{-t}\right)\left(c_{0}^{*}\left(U(0, t) x_{ \pm}(t)\right)\right)+c_{0}^{*}\left(U(0, t) x_{ \pm}(t)\right)
$$

The Equation ((10) with (11)) is our key problem for the solution of (1-5). If we can get the solution of sigma from (10) and (11), then we can easily find the solution $v$ from the following equation

$$
v\left(x_{ \pm}(t), t\right)=\sigma_{x}\left(x_{ \pm}(t), t\right) .
$$

\subsection{Gaussian Kernel}

First, consider initial condition problem

$$
\begin{cases}\sigma_{t}=\sigma_{x x}-\sigma & \text { in }\left(x_{-}<x<x_{+}\right) \times(0<t \leq T), \\ \sigma(0)=\sigma_{0} & \text { in }\left(l_{-} \leq x \leq l_{+}\right) .\end{cases}
$$

Now, we multiply $\mathrm{e}^{t}$ to both sides of (13), and take $\hat{\sigma}=\mathrm{e}^{t} \sigma$, then we have

$$
\begin{cases}\hat{\sigma}=\hat{\sigma}_{x x} & \text { in }\left(x_{-}<x<x_{+}\right) \times(0<t \leq T), \\ \hat{\sigma}(0)=\sigma_{0} & \text { in }\left(l_{-} \leq x \leq l^{+}\right),\end{cases}
$$

If $\hat{\sigma}$ defined in the whole domain $(-\infty,+\infty)$, then the solution of (14) would be

$$
\hat{\sigma}(x, t)=\int_{-\infty}^{+\infty} G(x-y, t) \sigma_{0}(y) \mathrm{d} y,
$$

where,

$$
G(x, t)=\frac{1}{2 \sqrt{\pi t}} \mathrm{e}^{-\frac{x^{2}}{4 t}}
$$

However, $\hat{\sigma}$ (or $\sigma$ ) is defined in bounded domain $\left(x_{-}(t), x_{+}(t)\right)$, therefore we cannot have the solution (15) for our case. But, at least we can see that the Gaussian function $G$ is differentiable against $x$ and $t$, and satisfies heat equation

$$
G_{t}(x, t)=G_{x x}(x, t) .
$$

Thus, we can say that $G$ is can be a fundamental solution for the heat equation.

Next, consider parameters $(\xi, \tau)$ in Gaussian function $G$. Define a new function

$$
U(x, t ; \xi, \tau)= \begin{cases}\frac{1}{2 \sqrt{\pi(t-\tau)}} \mathrm{e}^{-\frac{(x-\xi)^{2}}{4(t-\tau)}} & t>\tau, \\ 0 & t<\tau, \\ 0 & x \neq \xi, t=\tau\end{cases}
$$

$U$ is differentiable for all $x$ and t except $(x, t)=(\xi, \tau)$, and satisfies

$$
U_{x x}-U_{t}=0 \text {. }
$$

If we consider $U$ is the function of $(\xi, \tau)$ with parameters $(x, t)$, then $U$ is differentiable for all $\xi$ and $\tau$ except $(\xi, \tau)=(x, t)$, and satisfies 


$$
U_{\xi \xi}+U_{\tau}=0
$$

Note that

$$
\left\{\begin{array}{l}
U_{\xi}(x, t ; \xi, \tau)=-U_{x}(x, t ; \xi, \tau), \\
U_{\tau}(x, t ; \xi, \tau)=-U_{t}(x, t ; \xi, \tau), \\
U(x, t ;-\xi, \tau)=U(-x, t ; \xi, \tau) .
\end{array}\right.
$$

$U$ is also be a fundamental solution for the heat equation. Next we apply this fundamental solution $U$ to express the solution for the initial value problem (14).

\subsection{Green's Function}

In order to find the solution of (14), we need to introduce new function as follows

$$
\begin{aligned}
g(x, t ; \xi, \tau) & =U(x, t ; \xi, \tau)-U(x, t ;-\xi, \tau), \\
G(x, t ; \xi, \tau) & =U(x, t ; \xi, \tau)+U(x, t ;-\xi, \tau) .
\end{aligned}
$$

Clearly, we can see that $g$ and $G$ are differentiable for all $x$ and $t$ except $(x, t)=(\xi, \tau)$ and $(x, t)=(-\xi, \tau)$, and satisfies

$$
L g=g_{x x}-g_{t}=0, L G=0,
$$

and $g$ and $G$ are differentiable for all $\xi$ and $\tau$ except $(\xi, \tau)=(-x, t)$, therefore satisfies

$$
L^{*} g=g_{\xi \xi}+g_{\tau}=0, L^{*} G=0 .
$$

Since,

$$
L g=g_{x x}-g_{t}=U_{x x}(x, t ; \xi, \tau)-U_{x x}(x, t ;-\xi, \tau)-U_{t}(x, t ; \xi, \tau)+U_{t}(x, t ;-\xi, \tau),
$$

by (18), we can easily prove (23) and (24). Using the third property of $U$ in (20), we can get

$$
\begin{gathered}
\left.g\right|_{x=0}=\left.g\right|_{\xi=0}=0, \\
\left.G_{x}\right|_{x=0}=\left.G_{\xi}\right|_{\xi=0}=0,
\end{gathered}
$$

where, $g$ and $G$ are called Green's first type function and second type function, respectively.

\subsection{Free Moving Boundary Problem}

Now, we consider free moving boundary $x_{ \pm}=x_{ \pm}(t)$ as in Figure 4 with the following system:

$$
\begin{cases}\sigma_{t}=\sigma_{x x}-\sigma & \left(x_{-}(t)<x<x_{+}(t), 0<t \leq T\right), \\ \sigma\left(x_{ \pm}(t), t\right)=\sigma_{ \pm}(t) & (0<t \leq T), \\ \sigma(x, 0)=\sigma_{0}(x) \geq 0 & \left(l_{-}<x<l_{+}\right), \\ x_{ \pm}^{\prime}(t)=-\sigma_{x}\left(x_{ \pm}(t), t\right) & (0<t \leq T), \\ x_{ \pm}(0)=l_{ \pm} & \end{cases}
$$

where, 


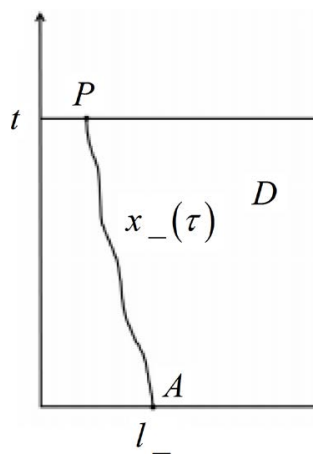

Figure 4. Free moving boundary of (27).

$$
\sigma_{ \pm}(t)=\left(1-\mathrm{e}^{-t}\right) c_{0}\left(U(0, t) x_{ \pm}(t)\right)+c_{0}^{*}\left(U(0, t) x_{ \pm}(t)\right) .
$$

Similar to (13), we have the following system

$$
\begin{cases}\hat{\sigma}_{t}=\hat{\sigma}_{x x} & \left(x_{-}(t)<x<x_{+}(t), 0<t \leq T\right), \\ \hat{\sigma}\left(x_{ \pm}(t), t\right)=\mathrm{e}^{t} \hat{\sigma}_{ \pm}(t) & (0<t \leq T), \\ \hat{\sigma}(x, 0)=\hat{\sigma}_{0}(x) \geq 0 & \left(l_{-}<x<l_{+}\right), \\ x_{ \pm}^{\prime}(t)=-\mathrm{e}^{-t} \hat{\sigma}_{x}\left(x_{ \pm}(t), t\right) & (0<t \leq T), \\ x_{ \pm}(0)=l_{ \pm}, & \end{cases}
$$

where,

$$
\hat{\sigma}(x, t)=\mathrm{e}^{t} \sigma(x, t) \text { in } D .
$$

Define a domain $D$ as (Figure 4)

$$
D=\left\{(\xi, \tau) \mid x_{-}(\tau)<\xi<x_{+}(\tau), 0<\tau<t\right\} .
$$

Take $\hat{\sigma}(\xi, \tau)$ as,

$$
\hat{\sigma}_{\xi \xi}-\hat{\sigma}_{\tau}=0 .
$$

Furthermore, from (24), we know

$$
g_{\xi \xi}+g_{\tau}=0
$$

in D. Combining (29) and (30), we have

$$
\iint_{D}\left[g\left(\hat{\sigma}_{\xi \xi}-\hat{\sigma}_{\tau}\right)-\hat{\sigma}\left(g_{\xi \xi}+g_{\tau}\right)\right] \mathrm{d} \xi \mathrm{d} \tau=0 .
$$

According to Green's formula, the left side of the Equation (31) becomes

$$
\begin{aligned}
& \iint_{D}\left[g\left(\hat{\sigma}_{\xi \xi}-\hat{\sigma}_{\tau}\right)-\hat{\sigma}\left(g_{\xi \xi}+g_{\tau}\right)\right] \mathrm{d} \xi \mathrm{d} \tau \\
& =\iint_{D} g \hat{\sigma}_{\xi \xi}-\hat{\sigma} g_{\xi \xi}-\left(\hat{\sigma}_{\tau} g+\hat{\sigma} g_{\tau}\right) \mathrm{d} \xi \mathrm{d} \tau \\
& =\int_{\overline{Q P}} \hat{\sigma} g \mathrm{~d} \xi+\int_{\overline{P A B Q}} \hat{\sigma} g \mathrm{~d} \xi+\left(g \hat{\sigma}_{\xi}-\hat{\sigma} g_{\xi}\right) \mathrm{d} \tau .
\end{aligned}
$$

Finally, we have

$$
\begin{aligned}
& \int_{\overline{P Q}} g(x, t+h ; \xi, t) \hat{\sigma}(\xi, t) \mathrm{d} \xi=\int_{\overline{P A B Q}} g(x, t+h ; \xi, \tau) \hat{\sigma}(\xi, \tau) \mathrm{d} \xi \\
& +\left[g(x, t+h ; \xi, \tau) \hat{\sigma}_{\xi}(\xi, \tau)-\hat{\sigma}(\xi, \tau) g_{\xi}(x, t+h ; \xi, \tau)\right] \mathrm{d} \tau,
\end{aligned}
$$

since, 


$$
\int_{\overline{Q P}} \hat{\sigma} g \mathrm{~d} \xi=-\int_{\overline{P Q}} \hat{\sigma} g \mathrm{~d} \xi
$$

When $h \rightarrow 0$, the left side of (32) equals to

$$
\lim _{h \rightarrow 0} \int_{x_{-}(t)}^{x_{+}(t)} U(x, t+h ; \xi, t) \hat{\sigma}(\xi, t) \mathrm{d} \xi-\lim _{h \rightarrow 0} \int_{x_{-}(t)}^{x_{+}(t)} U(x, t+h ;-\xi, t) \hat{\sigma}(\xi, t) \mathrm{d} \xi .
$$

\section{Main Results}

In this section we will state the main results, for convenience we will divide this section into two parts. In the first part, we will give three propositions and one theorem. Where, Proposition 1 and Proposition 2 are useful in proving Proposition 3, and Proposition 3 proves Theorem 1. Theorem 1 represents the solution of $\sigma$ in free boundary problem (27). In the second part, we will derive the solution of boundary velocity $v_{ \pm}(t)$ and boundary position $x_{ \pm}(t)$ by using condition (12) and Theorem 1.

\subsection{Solution for Free Boundary Problem}

In order to prove Theorem 1, we need to prove following three propositions.

Proposition 1. Suppose that, $\hat{\sigma}(x, t)$ is continuous for $x$ on $\overrightarrow{P Q}$, when $p(x, t+h) \rightarrow p_{0}(x, t)$, then

$$
\lim _{h \rightarrow 0} \frac{\hat{\sigma}(x, t)}{2 \sqrt{\pi h}} \int_{x_{-}-x}^{x_{+}-x} \mathrm{e}^{-\frac{\eta^{2}}{4 h}} \mathrm{~d} \eta=\hat{\sigma}(x, t)
$$

holds.

Proof. We consider the left side of (34),

$$
\frac{\hat{\sigma}(x, t)}{2 \sqrt{\pi h}} \int_{x_{-}-x}^{x_{+}-x} \mathrm{e}^{-\frac{\eta^{2}}{4 h}} \mathrm{~d} \eta=\frac{\hat{\sigma}(x, t)}{2 \sqrt{\pi h}} \int_{\frac{x_{-}-x}{2 \sqrt{h}}}^{\frac{x_{+}-x}{2 \sqrt{h}}} \mathrm{e}^{-\zeta^{2}} \mathrm{~d}(2 \sqrt{h} \zeta)=\frac{\hat{\sigma}(x, t)}{\sqrt{\pi}} \int_{\frac{x_{-}-x}{2 \sqrt{h}}}^{\frac{x_{+}-x}{2 \sqrt{h}}} \mathrm{e}^{-\zeta^{2}} \mathrm{~d} \zeta .
$$

Since,

$$
\lim _{h \rightarrow 0} \frac{x_{-}-x}{2 \sqrt{h}}=-\infty, \lim _{h \rightarrow 0} \frac{x_{+}-x}{2 \sqrt{h}}=+\infty,
$$

hence,

$$
\lim _{h \rightarrow 0} \frac{\hat{\sigma}(x, t)}{2 \sqrt{\pi h}} \int_{x_{-}-x}^{x_{+}-x} \mathrm{e}^{-\frac{\eta^{2}}{4 h}} \mathrm{~d} \eta=\frac{\hat{\sigma}(x, t)}{\sqrt{\pi}} \int_{-\infty}^{+\infty} \mathrm{e}^{-\zeta^{2}} \mathrm{~d} \zeta=\hat{\sigma}(x, t) .
$$

This completes the proof of Proposition 1.

Proposition 2. Suppose that, $\hat{\sigma}(x, t)$ is continuous for $x$ on $\overrightarrow{P Q}$, when $p(x, t+h) \rightarrow p_{0}(x, t)$, then

$$
\lim _{h \rightarrow 0} \int_{x_{-}-x}^{x_{+}-x}[\hat{\sigma}(\eta+x, t)-\hat{\sigma}(x, t)] \mathrm{e}^{-\frac{\eta^{2}}{4 h}} \mathrm{~d} \eta \equiv 0
$$

holds.

Proof. We consider the left side of (35),

$$
\int_{x_{-}-x}^{x_{+}-x}[\hat{\sigma}(\eta+x, t)-\hat{\sigma}(x, t)] \mathrm{e}^{-\frac{\eta^{2}}{4 h}} \mathrm{~d} \eta \leq H_{1}+H_{2}+H_{3},
$$

where, 


$$
\begin{aligned}
& H_{1}=\int_{x_{-}-x}^{-\varepsilon}[\hat{\sigma}(\eta+x, t)-\hat{\sigma}(x, t)] \mathrm{e}^{-\frac{\eta^{2}}{4 h}} \mathrm{~d} \eta, \\
& H_{2}=\int_{-\varepsilon}^{\varepsilon}[\hat{\sigma}(\eta+x, t)-\hat{\sigma}(x, t)] \mathrm{e}^{-\frac{\eta^{2}}{4 h}} \mathrm{~d} \eta, \\
& H_{3}=\int_{\varepsilon}^{x_{+}-x}[\hat{\sigma}(\eta+x, t)-\hat{\sigma}(x, t)] \mathrm{e}^{-\frac{\eta^{2}}{4 h}} \mathrm{~d} \eta .
\end{aligned}
$$

Since $\varepsilon$ is small enough, then, we have

$$
\lim _{h \rightarrow 0} H_{2}=0 \text {. }
$$

Next, from the continuity of $\hat{\sigma}(x)$, for any small $\delta$, there exists $\gamma\left(0<\gamma<x_{+}-x\right)$, for $\varepsilon<\eta<x_{+}-x$, we have

$$
|\hat{\sigma}(\eta+x, t)-\hat{\sigma}(x, t)|<\delta,
$$

therefore,

$$
\begin{aligned}
\left|H_{3}\right| & =\left|\int_{\varepsilon}^{x_{+}-x}[\hat{\sigma}(\eta+x, t)-\hat{\sigma}(x, t)] \mathrm{e}^{-\frac{\eta^{2}}{4 h}} \mathrm{~d} \eta\right| \\
& \leq \int_{\varepsilon}^{x_{+}-x} \mathrm{e}^{-\frac{\eta^{2}}{4 h}} \mathrm{~d} \eta \leq \mathrm{e}^{-\frac{\varepsilon^{2}}{4 h}}\left(x_{+}-x-\varepsilon\right) \rightarrow 0(h \rightarrow 0) .
\end{aligned}
$$

Similarly we can get

$$
\lim _{h \rightarrow 0}\left|H_{1}\right|=0
$$

Therefore, (35) follows.

Proposition 3. Suppose that, $\hat{\sigma}(x, t)$ is continuous for $x$ on $\overrightarrow{P Q}$ and when $p(x, t+h) \rightarrow p_{0}(x, t)$, then

$$
\begin{aligned}
& \lim _{h \rightarrow 0} \int_{x_{-}(t)}^{x_{+}(t)} U(x, t+h ; \xi, t) \hat{\sigma}(\xi, t) \hat{\sigma}(\xi, t) \mathrm{d} \xi \\
& =\hat{\sigma}(x, t) .
\end{aligned}
$$

Proof. From the assumption of Proposition 3, we have

$$
\begin{aligned}
& \int_{x_{-}(t)}^{x_{+}(t)} U(x, t+h ; \xi, t) \hat{\sigma}(\xi, \tau) \mathrm{d} \xi \\
& =\int_{x_{-}-x}^{x_{+}-x} U(x, t+h ; \eta+x, t) \hat{\sigma}(\eta+x, t) \mathrm{d} \eta \\
& =\int_{x_{-}-x}^{x_{+}-x} \frac{\hat{\sigma}(\eta+x, \tau)}{2 \sqrt{\pi(t+h-t)}} \mathrm{e}^{-\frac{\eta^{2}}{4(t+h-t)}} \mathrm{d} \eta \\
& =\frac{1}{2 \sqrt{\pi h}} \int_{x_{-}-x}^{x_{+}-x} \hat{\sigma}(\eta+x, t) \mathrm{e}^{-\frac{\eta^{2}}{4 h}} \mathrm{~d} \eta \\
& =\frac{1}{2 \sqrt{\pi h}} \int_{x_{-}-x}^{x_{+}-x} \mathrm{e}^{-\frac{\eta^{2}}{4 h}} \mathrm{~d} \eta+\frac{1}{2 \sqrt{\pi h}} \int_{x_{-}-x}^{x_{+}-x}[\hat{\sigma}(\eta+x, t)-\hat{\sigma}(x, t)] \mathrm{e}^{-\frac{\eta^{2}}{4 h}} \mathrm{~d} \eta .
\end{aligned}
$$

By Proposition 1 and Proposition 2, we can prove Proposition 3. This completes the proof.

Theorem 1. Suppose that $\hat{\sigma}(x, t)$ is continuous for $x$ on $\overrightarrow{P Q}$, then we have 


$$
\begin{aligned}
\sigma(x, t)= & \mathrm{e}^{-t}\left[\int_{L_{-}}^{l_{+}} g(x, t ; \xi, 0) \sigma_{0}(\xi) \mathrm{d} \xi-\int_{0}^{t} g\left(x, t ; x_{-}(\tau), \tau\right) \mathrm{e}^{\tau} v_{-}(\tau) \mathrm{d} \tau\right. \\
& +\int_{0}^{t} g_{\xi}\left(x, t ; x_{-}(\tau), \tau\right) \mathrm{e}^{\tau} \sigma_{-}(\tau) \mathrm{d} \tau+\int_{0}^{t} g\left(x, t ; x_{+}(\tau), \tau\right) \mathrm{e}^{\tau} v_{+}(\tau) \mathrm{d} \tau \\
& \left.-\int_{0}^{t} g_{\xi}\left(x, t ; x_{+}(\tau), \tau\right) \mathrm{e}^{\tau} \sigma_{+}(\tau) \mathrm{d} \tau\right] .
\end{aligned}
$$

Proof. Proposition 3 gives the calculation of the first term of form (33). Regarding to the second term of (33), we are using the third property of $U$ in (20),

$$
\int_{x_{-}(t)}^{x_{+}(t)} U(x, t+h ;-\xi, t) \hat{\sigma}(\xi, t) \mathrm{d} \xi=\int_{x_{-}(t)}^{x_{+}(t)} U(-x, t+h ; \xi, t) \hat{\sigma}(\xi, t) \mathrm{d} \xi,
$$

for $x_{-}(t)<\xi<x_{+}(t)$. Similarly to (36), we have

$$
\lim _{h \rightarrow 0} \int_{x_{-}(t)}^{x_{+}(t)} U(-x, t+h ; \xi, t) \hat{\sigma}(\xi, t) \mathrm{d} \xi=\hat{\sigma}(-x, t),
$$

since $-x$ not belongs to $x_{-}(t)<\xi<x_{+}(t)$, therefore, $\hat{\sigma}(-x, t) \equiv 0$, and the second term of (33) equivalent to 0 .

The above results show that the left side of Equation (32) satisfies

$$
\lim _{h \rightarrow 0} \int_{\overline{P Q}} g(x, t+h ; \xi, t) \hat{\sigma}(\xi, t) \mathrm{d} \xi=\hat{\sigma}(x, t) .
$$

Then, we have

$$
\begin{aligned}
\hat{\sigma}(x, t)= & \lim _{h \rightarrow 0} \int_{\frac{P A B Q}{P}} g(x, t+h ; \xi, \tau) \hat{\sigma}(\xi, \tau) \mathrm{d} \xi \\
& +\left[g(x, t+h ; \xi, \tau) \hat{\sigma}_{\xi}(\xi, \tau)-\hat{\sigma}(\xi, \tau) g_{\xi}(x, t+h ; \xi, \tau)\right] \mathrm{d} \tau .
\end{aligned}
$$

For the right side of (39), it is clear that point $(x, t+h)$ is separated from $\overrightarrow{P A B Q}$, and $g(x, t+h ; \xi, \tau)$ and $\hat{\sigma}(\xi, \tau)$ is continuous for $h$ on $\overrightarrow{P A B Q}$, since,

$$
\begin{aligned}
& \lim _{h \rightarrow 0} U(x, t+h ; \xi, \tau) \hat{\sigma}(\xi, \tau) \\
& =\lim _{h \rightarrow 0}\left(\frac{\hat{\sigma}(\xi, \tau)}{2 \sqrt{\pi(t+h-\tau)}} \mathrm{e}^{-\frac{(x-\xi)^{2}}{4(t+h-\tau)}}\right)=\frac{\hat{\sigma}(\xi, \tau)}{2 \sqrt{\pi(t-\tau)}} \mathrm{e}^{-\frac{(x-\xi)^{2}}{4(t-\tau)}} \\
& =U(x, t ; \xi, \tau) \hat{\sigma}(\xi, \tau),
\end{aligned}
$$

and

$$
\begin{aligned}
\lim _{h \rightarrow 0} g(x, t+h ; \xi, \tau) & =\lim _{h \rightarrow 0} U(x, t+h ; \xi, \tau)-\lim _{h \rightarrow 0} U(x, t+h ;-\xi, \tau) \\
& =U(x, t ; \xi, \tau)-U(x, t ;-\xi, \tau)=g(x, t ; \xi, \tau) .
\end{aligned}
$$

Moreover, note $g$ is continuous for all $\xi$ and $\tau$ on $\overrightarrow{P A B Q}$. Hence from the bounded convergence theorem we have

$$
\begin{aligned}
& \lim _{h \rightarrow 0} \int_{\frac{P A B Q}{P}} g(x, t+h ; \xi, \tau) \hat{\sigma}(\xi, \tau) \mathrm{d} \xi+\left[g(x, t+h ; \xi, \tau) \hat{\sigma}_{\xi}-\hat{\sigma} g_{\xi}(x, t+h ; \xi, \tau)\right] \mathrm{d} \tau \\
& =\int_{\frac{P A B Q}{}} g(x, t ; \xi, \tau) \hat{\sigma}(\xi, \tau) \mathrm{d} \xi+\left[g(x, t ; \xi, \tau) \hat{\sigma}_{\xi}-\hat{\sigma} g_{\xi}(x, t ; \xi, \tau)\right] \mathrm{d} \tau \\
& \quad \text { for }\left(x_{-}(t)<x<x_{+}(t)\right) . \text { Now, take together }(38) \text { and }(40), \text { we have } \\
& \quad \sigma(x, t)=\int_{\overline{P A B Q}} g(x, t ; \xi, \tau) \hat{\sigma}(\xi, \tau) \mathrm{d} \xi+\left[g(x, t ; \xi, \tau) \hat{\sigma}_{\xi}-\hat{\sigma} g_{\xi}(x, t ; \xi, \tau)\right] \mathrm{d} \tau .
\end{aligned}
$$

Equation (41) is useful to prove Theorem 1, which can be written as, 


$$
\begin{aligned}
\sigma(x, t)= & \mathrm{e}^{-t}\left[\int_{l_{-}}^{l_{+}} g(x, t ; \xi, 0) \sigma_{0}(\xi) \mathrm{d} \xi-\int_{0}^{t} g\left(x, t ; x_{-}(\tau), \tau\right) \mathrm{e}^{\tau} v_{-}(\tau) \mathrm{d} \tau\right. \\
& +\int_{0}^{t} g_{\xi}\left(x, t ; x_{-}(\tau), \tau\right) \mathrm{e}^{\tau} \sigma_{0}(\tau) \mathrm{d} \tau+\int_{0}^{t} g\left(x, t ; x_{+}(\tau), \tau\right) \mathrm{e}^{\tau} v_{+}(\tau) \mathrm{d} \tau \\
& \left.-\int_{0}^{t} g_{\xi}\left(x, t ; x_{+}(\tau), \tau\right) \mathrm{e}^{\tau} \sigma_{+}(\tau) \mathrm{d} \tau\right] .
\end{aligned}
$$

This completes the proof of Theorem 1.

\subsection{Solution for Velocity}

In the above result (37) in Theorem 1, all variables are known except $\sigma_{\xi}\left(x_{ \pm}(\tau), \tau\right)$. From the Equation (6) and last condition of (28), we can write

$$
\begin{gathered}
x_{ \pm}^{\prime}(t)=-v_{ \pm}(t), \\
\lim _{x \rightarrow x_{ \pm}(t) \mp 0} \sigma_{x}(x, t)=v_{ \pm}(t),
\end{gathered}
$$

where, $v_{ \pm}(t)=v\left(x_{ \pm}(t), t\right)$ is velocity of $x_{ \pm}(t)$. To define $v_{ \pm}(t)$ we first differentiate the both side of (42), thereupon,

$$
\begin{aligned}
\sigma_{x}(x, t)= & \mathrm{e}^{-t}\left[\int_{L_{-}}^{l_{+}} g_{x}(x, t ; \xi, 0) \sigma_{0}(\xi) \mathrm{d} \xi-\int_{0}^{t} g_{x}\left(x, t ; x_{-}(\tau), \tau\right) \mathrm{e}^{\tau} v_{-}(\tau) \mathrm{d} \tau\right. \\
& +\int_{0}^{t} g_{\xi x}\left(x, t ; x_{-}(\tau), \tau\right) \mathrm{e}^{\tau} \sigma_{-}(\tau) \mathrm{d} \tau+\int_{0}^{t} g_{x}\left(x, t ; x_{+}(\tau), \tau\right) \mathrm{e}^{\tau} v_{+}(\tau) \mathrm{d} \tau \\
& \left.-\int_{0}^{t} g_{\xi x}\left(x, t ; x_{+}(\tau), \tau\right) \mathrm{e}^{\tau} \sigma_{+}(\tau) \mathrm{d} \tau\right]
\end{aligned}
$$

From the definition of $U(x, t ; \xi, \tau)$ one can easily get

$$
U_{x}(x, t ; \xi, \tau)=-U(\xi, t ; \xi, \tau),
$$

then,

$$
\begin{aligned}
g_{x}(x, t ; \xi, \tau) & =U_{x}(x, t ; \xi, \tau)-U_{x}(x, t ;-\xi, \tau) \\
& =-U_{\xi}(x, t ; \xi, \tau)+U_{\xi}(x, t ;-\xi, \tau) \\
& =-\frac{\partial}{\partial \xi}[U(x, t ; \xi, \tau)+U(x, t ;-\xi, \tau)] \\
& =-G_{\xi}(x, t ; \xi, \tau) .
\end{aligned}
$$

If we use above property, (45) becomes,

$$
\begin{aligned}
\sigma_{x}(x, t)= & -\mathrm{e}^{-t}\left[\int_{L_{-}}^{l_{+}} G_{\xi}(x, t ; \xi, 0) \sigma_{0}(\xi) \mathrm{d} \xi-\int_{0}^{t} G_{\xi}\left(x, t ; x_{-}(\tau), \tau\right) e^{\tau} v_{-}(\tau) \mathrm{d} \tau\right. \\
& +\int_{0}^{t} G_{\xi \xi}\left(x, t ; x_{-}(\tau), \tau\right) \mathrm{e}^{\tau} \sigma_{-}(\tau) \mathrm{d} \tau+\int_{0}^{t} G_{\xi}\left(x, t ; x_{+}(\tau), \tau\right) \mathrm{e}^{\tau} v_{+}(\tau) \mathrm{d} \tau \\
& \left.-\int_{0}^{t} G_{\xi \xi}\left(x, t ; x_{+}(\tau), \tau\right) \mathrm{e}^{\tau} \sigma_{+}(\tau) \mathrm{d} \tau\right] .
\end{aligned}
$$

Next, from (23), we have $G_{\xi \xi}=-G_{\tau}$, thus, (47) becomes

$$
\begin{aligned}
\sigma_{x}(x, t)= & -\mathrm{e}^{-t}\left[\int_{L_{-}}^{l_{+}} G_{\xi}(x, t ; \xi, 0) \sigma_{0}(\xi) \mathrm{d} \xi-\int_{0}^{t} G_{\xi}\left(x, t ; x_{-}(\tau), \tau\right) \mathrm{e}^{\tau} v_{-}(\tau) \mathrm{d} \tau\right. \\
& +\int_{0}^{t} G_{\tau}\left(x, t ; x_{-}(\tau), \tau\right) \mathrm{e}^{\tau} \sigma_{-}(\tau) \mathrm{d} \tau+\int_{0}^{t} G_{\xi}\left(x, t ; x_{+}(\tau), \tau\right) \mathrm{e}^{\tau} v_{+}(\tau) \mathrm{d} \tau \\
& \left.-\int_{0}^{t} G_{\tau}\left(x, t ; x_{+}(\tau), \tau\right) \mathrm{e}^{\tau} \sigma_{+}(\tau) \mathrm{d} \tau\right] .
\end{aligned}
$$

When $x$ tends to $x_{-}(t)$ from the left, 


$$
\begin{aligned}
v_{-}(t)= & -\mathrm{e}^{-t}\left[\int_{L_{-}}^{l_{+}} G_{\xi}\left(x_{-}(t), t ; \xi, 0\right) \sigma_{0}(\xi) \mathrm{d} \xi\right. \\
& -\left\{\int_{0}^{t} G_{\xi}\left(x_{-}(t), t ; x_{-}(\tau), \tau\right) \mathrm{e}^{\tau} v_{-}(\tau) \mathrm{d} \tau+\frac{1}{2} \mathrm{e}^{t} v_{-}(t)\right\} \\
& +\int_{0}^{t} G_{\tau}\left(x_{-}(t), t ; x_{-}(\tau), \tau\right) \mathrm{e}^{\tau} \sigma_{-}(\tau) \mathrm{d} \tau \\
& +\int_{0}^{t} G_{\xi}\left(x_{-}(t), t ; x_{+}(\tau), \tau\right) \mathrm{e}^{\tau} v_{+}(\tau) \mathrm{d} \tau \\
& \left.+\int_{0}^{t} G_{\tau}\left(x_{-}(t), t ; x_{+}(\tau), \tau\right) \mathrm{e}^{\tau} \sigma_{+}(\tau) \mathrm{d} \tau\right]
\end{aligned}
$$

(See appendix for specific information).

$$
\begin{aligned}
v_{-}(t)= & -\frac{2}{3} \mathrm{e}^{-t}\left[\int_{L_{-}}^{l_{+}} G_{\xi}\left(x_{-}(t), t ; \xi, 0\right) \sigma_{0}(\xi) \mathrm{d} \xi-\int_{0}^{t} G_{\xi}\left(x_{-}(t), t ; x_{-}(\tau), \tau\right) \mathrm{e}^{\tau} v_{-}(\tau) \mathrm{d} \tau\right. \\
& +\int_{0}^{t} G_{\tau}\left(x_{-}(t), t ; x_{-}(\tau), \tau\right) \mathrm{e}^{\tau} \sigma_{-}(\tau) \mathrm{d} \tau+\int_{0}^{t} G_{\xi}\left(x_{-}(t), t ; x_{+}(\tau), \tau\right) \mathrm{e}^{\tau} v_{+}(\tau) \mathrm{d} \tau \\
& \left.+\int_{0}^{t} G_{\tau}\left(x_{-}(t), t ; x_{+}(\tau), \tau\right) \mathrm{e}^{\tau} \sigma_{+}(\tau) \mathrm{d} \tau\right] . \\
& \text { When } x \rightarrow x_{+}(t)-0, \\
v_{-}(t)= & -2 \mathrm{e}^{-t}\left[\int_{L_{-}}^{l_{+}} G_{\xi}\left(x_{+}(t), t ; \xi, 0\right) \sigma_{0}(\xi) \mathrm{d} \xi-\int_{0}^{t} G_{\xi}\left(x_{+}(t), t ; x_{-}(\tau), \tau\right) \mathrm{e}^{\tau} v_{-}(\tau) \mathrm{d} \tau\right. \\
& +\int_{0}^{t} G_{\tau}\left(x_{+}(t), t ; x_{-}(\tau), \tau\right) \mathrm{e}^{\tau} \sigma_{-}(\tau) \mathrm{d} \tau+\int_{0}^{t} G_{\xi}\left(x_{+}(t), t ; x_{+}(\tau), \tau\right) \mathrm{e}^{\tau} v_{+}(\tau) \mathrm{d} \tau \\
& \left.+\int_{0}^{t} G_{\tau}\left(x_{+}(t), t ; x_{+}(\tau), \tau\right) \mathrm{e}^{\tau} \sigma_{+}(\tau) \mathrm{d} \tau\right] .
\end{aligned}
$$

Integrate both sides of (43) from 0 to $t$, we have

$$
\int_{0}^{t} x_{ \pm}^{\prime}(t) \mathrm{d} t=-\int_{0}^{t} v_{ \pm}(t) \mathrm{d} t
$$

therefore, we have

$$
x_{ \pm}(t)=l_{ \pm}-\int_{0}^{t} v_{ \pm}(t) \mathrm{d} t .
$$

Refer appendix for specific information about how (49) and (51) are followed by (48) as $x \rightarrow x_{-}(t)+0$ and $x \rightarrow x_{+}(t)-0$, respectively.

\section{Conclusions}

Actin filaments are cytoskeleton in cytoplasm, which can drive cell deformation, migration [14], and even invasion to the surrounding matrix [15]. How actin filaments are driving cancer cell invasion has been discussed in [1]. They considered four particles, actins, ECMs, MMPs and ECM fragments, where actins and MMPs act in intracellular area, ECMs and ECM fragments exist in extracellular area. Although, they had an excellent result which can describe the deformation of the cell membrane; however, they cannot control the actins, which should not be in extracellular area, diffused throughout the whole domain. To improve the work in [1], in this paper we added a free-boundary $\Gamma$, which is defined as (3), to separate the whole domain into two sub-domains, intracellular domain and extracellular domain. Where, the free-boundary $\Gamma$ is proportional to the cell membrane, which is considered to be pushed by actin assembly ( $n$ ), and hence we took the boundary velocity $v$ depending on the assembly rate of actin pro- 
teins.

Colin et al [16] introduced a model to describe endothelial cells' migration on bioactive micro-patterned polymers. In their model, the chemotaxis term is considered as cell-cell interaction, therefore, they considered two domains, the adhesive domain and the non-adhesive domain, where adhesive areas are surrounded by non-adhesive areas. By the motivation of the work in [16], we divided the domain into two parts; however, because of the high complexity of the model, it is difficult to deal with the solution. Thus, we simplified the model and turn the problem into Stefan problem (27), and then consider its solution in one-dimensional case. As a result, integral equations $x_{ \pm}(t)$ and $v_{ \pm}(t)$ were obtained. Therefore, problem of solving system (27) was turned to the problem of solving combination of (50), (51) and (52). The results are useful because the system (27) became more suitable to apply finite difference method or other methods, for example, Picard's successive method. On the other hand, from the biological point of view, the results, in this paper, are not enough to explain the biological meaning; however, they will lead us to further step to discuss the solution of the modified model (1 - 5).

We have more interesting topics which deserve further investigations, such as numerical simulations of the integral equations $(48,50-52)$ and how we can get the solution of the original modified model $(1-4)$ based on integral solution.

\section{Acknowledgements}

This work was supported by the National Natural Science Foundation of China (Grant Nos.11401509) and the Natural Science Foundation of Xinjiang University (Starting Fund for Doctors, Grant No. BS130102).

\section{Conflict of Interests Statements}

All authors of this article declare: there is no conflict of interests regarding the publication of this article.

\section{References}

[1] Saito, T., Rouzimaimaiti, M., Koshikawa, N., Seiki, M., Ichikawa, K. and Suzuki, T. (2012) Mathematical Modeling of Invadopodia Formation. Journal of Theoretical Biology, 298, 138-146. https://doi.org/10.1016/j.jtbi.2011.12.018

[2] Shih, T. and Lindley, C. (2006) Bevacizumab: An Angiogenesis Inhibitor for the Treatment of Solid Malignancies. Clinical Therapeutics, 28, 1779-1802. https://doi.org/10.1016/j.clinthera.2006.11.015

[3] Birkedal-Hansen, H., Moore, W.G., Bodden, M.K., Windsor, L.J., Birkedal-Hansen, B., De-Carlo, A. and Engler, J.A. (1993) Matrix Metalloproteinases: A Review. Critical Reviews in Oral Biology \& Medicine, 4, 197-250. https://doi.org/10.1177/10454411930040020401

[4] Niiya, D., Egawa, N., Sakamoto, T., Kikkawa, Y., Shinkawa, T., Isobe, T., Koshikawa, N. and Seiki, M. (2009) Identification and Characterization of Lutheran Blood Group Glycoprotein as a New Substrate of Membrane-Type 1 Matrix Metalloproteinase 1 (MT1-MMP): A Systemic Whole Cell Analysis of MT1-MMP-Associating Proteins in A431 Cells. Journal of Biological Chemistry, 284, 27360-27369. 
https://doi.org/10.1074/jbc.M109.029124

[5] Sakurai-Yageta, M., Recchi, C., Le Dez, G., Sibarita, J.B., Daviet, L., Camonis, J., D'Souza-Schorey, C. and Chavrier, P. (2008) The Interaction of IQGAP1 with the Exocyst Complex Is Required for Tumor Cell Invasion Downstream of Cdc42 and RhoA. Journal of Cell Biology, 181, 985-998. https://doi.org/10.1083/jcb.200709076

[6] Koshikawa, N., Minegishi, T., Sharabi, A., Quaranta, V. and Seiki, M. (2005). Membrane-Type Matrix Metalloproteinase-1 (MT1-MMP) Is a Processing Enzyme for Human Laminin Gamma2 Chain. Journal of Biological Chemistry, 280, 88-93. https://doi.org/10.1074/jbc.M411824200

[7] Chaplain, M.A. and Anderson, A.R. (2003) Mathematical Modelling of Tissue Invasion. In: Preziosi, L., Ed., Cancer Modelling and Simulation, Chapman and Hall/CRC, London, 269-298. https://doi.org/10.1201/9780203494899.ch10

[8] Anderson, A.R.A. (2005). A Hybrid Mathematical Model of Solid Tumor Invasion: The Importance of Cell Adhesion. Mathematical Medicine and Biology, 22, 163 186. https://doi.org/10.1093/imammb/dqi005

[9] Enderling, H., Alexander, N.R., Clark, E.S., Branch, K.M., Estrada, L., Anderson, A.R.A. and Weaver, A.M. (2008) Dependence of Invadopodia Function on Collagen Fiber Spacing and Cross-Linking: Computational Modeling and Experimental Evidence. Biophysical Journal, 95, 2203-2218.

https://doi.org/10.1529/biophysj.108.133199

[10] Konstantin, E.S. and Alexander, P.K. (2014) On the Global Dynamics of One Cancer Tumor Growth Model. Communications in Nonlinear Science and Numerical Simulation, 19, 1486-1495. https://doi.org/10.1016/j.cnsns.2013.09.023

[11] Mehmet, I. and Stephen, P.B. (2010) Chaos in a Three-Dimensional Cancer Model. International Journal of Bifurcation and Chaos, 20, 71-79. https://doi.org/10.1142/S0218127410025417

[12] Campellone, K.G. and Welch, M.D. (2010). A Nucleator Arms Race: Cellular Control of Actin Assembly. Nature Reviews Molecular Cell Biology, 11, 237-251. https://doi.org/10.1038/nrm2867

[13] Reenstra, W.R., Orlow, D.L. and Svoboda, K.K.H. (2002). ECM-Stimulated Signaling and Actin Reorganization in Embryonic Corneal Epithelia Are Rho Dependent. Investigative Ophthalmology \& Visual Science, 43, 3181-3189.

[14] Pollard, T.D. and Borisy, G.G. (2003). Cellular Motility Driven by Assembly and Disassembly of Actin Filaments. Cell, 112, 453-465.

https://doi.org/10.1016/S0092-8674(03)00120-X

[15] Nurnberg, A., Kitzing, T. and Grosse, R. (2011) Nucleating Actin for Invasion. Nature Reviews Cancer, 11, 177-187. https://doi.org/10.1038/nrc3003

[16] Colin, T., Durrieu, M.C., Joie, J., Lei, Y., Mammeri, Y., Poignard, C. and Saut, O. (2013) Modelling of the Migration of Endothelial Cells on Bioactive Micropatterned Polymers. Mathematical Biosciences and Engineering, 10, 997-1015.

https://doi.org/10.3934/mbe.2013.10.997 


\section{Appendix}

This appendix provides specific information about how we get (49) from (48).

a) Confirmation of

$$
\lim _{x \rightarrow x_{-}(t)+0} \int_{L_{-}}^{l_{+}} G_{\xi}(x, t ; \xi, 0) \sigma_{0}(\xi) \mathrm{d} \xi=\int_{l_{-}}^{l_{+}} G_{\xi}\left(x_{-}(t), t ; \xi, 0\right) \sigma_{0}(\xi) \mathrm{d} \xi,
$$

where, $\sigma_{0}(\xi)$ is continuous in closed interval $\left[l_{-}, l_{+}\right]$.

Proof. From the definition of $G$, we have

$\int_{l_{-}}^{l_{+}} G_{\xi}(x, t ; \xi, 0) \sigma_{0}(\xi) \mathrm{d} \xi=\int_{l_{-}}^{l_{+}} U_{\xi}(x, t ; \xi, 0) \sigma_{0}(\xi) \mathrm{d} \xi-\int_{l_{-}}^{l_{+}} U_{\xi}(x, t ;-\xi, 0) \sigma_{0}(\xi) \mathrm{d} \xi$.

Then, from the definition of $U$,

$$
\int_{l_{-}}^{l_{+}} U_{\xi}(x, t ; \xi, 0) \sigma_{0}(\xi) \mathrm{d} \xi=\frac{1}{4 \sqrt{\pi t^{3}}} \int_{l_{-}}^{l_{+}} \sigma_{0}(\xi)(x-\xi) \mathrm{e}^{-\frac{(x-\xi)^{2}}{4 t}} \mathrm{~d} \xi,
$$

where, $\sigma_{0}(\xi)$ is continuous in $\left[l_{-}, l_{+}\right]$, therefore, it is clear that $\sigma_{0}(\xi)(x-\xi) \mathrm{e}^{-\frac{(x-\xi)^{2}}{4 t}}$ is continuous on $\forall \xi \in\left[l_{-}, l_{+}\right]$. Now, we prove there exist $g(\xi)$ such that $\left|\frac{1}{4 \sqrt{\pi t^{3}}} \sigma_{0}(\xi)(x-\xi) \mathrm{e}^{-\frac{(x-\xi)^{2}}{4 t}}\right| \leq g(\xi)$.

For further calculations, we introduce inequality

$$
\mathrm{e}^{-R^{2}}<\varepsilon|R|^{-a}
$$

where, $R \rightarrow \infty$, and $a$ is positive constant. We can prove (54) by

$$
\lim _{R \rightarrow \infty} \frac{\mathrm{e}^{-R^{2}}}{R^{-a}}=0,
$$

then, by the definition of Function-limit, we have for any $\varepsilon>0$, and

$$
\left|\frac{\mathrm{e}^{-R^{2}}}{R^{-a}}-0\right|<\varepsilon
$$

then,

$$
\mathrm{e}^{-R^{2}}<\varepsilon\left|R^{-a}\right|
$$

Therefore, applying (54) we can get

$$
\left|\frac{1}{4 \sqrt{\pi t^{3}}} \sigma_{0}(\xi)(x-\xi) \mathrm{e}^{-\frac{(x-\xi)^{2}}{4 t}}\right| \leq \frac{\left|\sigma_{0}(\xi)\right||x-\xi|}{4 \sqrt{\pi t^{3}}}\left|\frac{2 \sqrt{t}}{x-\xi}\right|=\frac{\left|\sigma_{0}(\xi)\right|}{2 t \sqrt{\pi}} .
$$

Take $g(\xi)=\frac{\left|\sigma_{0}(\xi)\right|}{2 t \sqrt{\pi}}$, from the continuity of $\sigma_{0}(\xi)$ in closed set $\left[l_{-}, l_{+}\right]$we can say $\sigma_{0}(\xi)$ is bounded, and then there exist $M>0$, such that

$$
\left|\sigma_{0}(\xi)\right|<M
$$

Hence, we have

$$
\left|\int_{l_{-}}^{l_{+}} \frac{1}{4 \sqrt{\pi t^{3}}} \sigma_{0}(\xi)(x-\xi) \mathrm{e}^{-\frac{(x-\xi)^{2}}{4 t}} \mathrm{~d} \xi\right| \leq \frac{M}{2 t \sqrt{\pi}} \int_{L_{-}}^{l_{+}} \mathrm{d} \xi=\frac{M}{2 t \sqrt{\pi}}\left(l_{+}-l_{-}\right),
$$


which implies that $\frac{1}{4 \sqrt{\pi t^{3}}} \sigma_{0}(\xi)(x-\xi) \mathrm{e}^{-\frac{(x-\xi)^{2}}{4 t}}$ is integrable for all $x \in\left[l_{-}, l_{+}\right]$ and continuous for all $\xi \in\left[l_{-}, l_{+}\right]$. Finally applying continuity of integrals theorem, it follows that

$$
\begin{aligned}
& \lim _{x \rightarrow \chi_{-}(t)+0} \frac{1}{4 \sqrt{\pi t^{3}}} \int_{L_{-}}^{I_{+}} \sigma_{0}(\xi)(x-\xi) \mathrm{e}^{-\frac{(x-\xi)^{2}}{4 t}} \mathrm{~d} \xi \\
& =\frac{1}{4 \sqrt{\pi t^{3}}} \int_{L_{-}}^{l_{+}} \sigma_{0}(\xi)\left(x_{-}(t)-\xi\right) \mathrm{e}^{-\frac{(x-\xi)^{2}}{4 t}} \mathrm{~d} \xi .
\end{aligned}
$$

On the other hand,

$$
\lim _{x \rightarrow x_{-}(t)+0} \int_{L_{-}}^{l_{+}} U_{\xi}(x, t ; \xi, 0) \sigma_{0}(\xi) \mathrm{d} \xi=\int_{L_{-}}^{l_{+}} U_{\xi}\left(x_{-}(t), t ; \xi, 0\right) \sigma_{0}(\xi) \mathrm{d} \xi .
$$

Similarly, we can get

$$
\lim _{x \rightarrow x_{-}(t)+0} \int_{L_{-}}^{l_{+}} U_{\xi}(x, t ;-\xi, 0) \sigma_{0}(\xi) \mathrm{d} \xi=\int_{L_{-}}^{I_{+}} U_{\xi}\left(x_{-}(t), t ;-\xi, 0\right) \sigma_{0}(\xi) \mathrm{d} \xi .
$$

b) confirmation of

$$
\begin{aligned}
& \lim _{x \rightarrow x_{-}(t)+0} \int_{0}^{t} G_{\xi}\left(x, t ; x_{-}(\tau), \tau\right) \hat{\sigma}_{\xi}\left(x_{-}(\tau), \tau\right) \mathrm{d} \tau \\
& =\int_{0}^{t} G_{\xi}\left(x_{-}(t), t ; x_{-}(\tau), \tau\right) \hat{v}_{-}(\tau) \mathrm{d} \tau-\frac{1}{2} \hat{v}_{-}(t) .
\end{aligned}
$$

Proof. Set

$$
v(x, t)=\int_{0}^{t} U\left(x, t ; x_{-}(\tau), \tau\right) \hat{\sigma}_{\xi}\left(x_{-}(\tau), \tau\right) \mathrm{d} \tau,
$$

if $\hat{\sigma}_{\xi}\left(x_{-}(\tau), \tau\right)$ is bounded in $(0, t)$, then,

$$
\lim _{x \rightarrow x_{-}(t)+0} v(x, t)=v\left(x_{-}(t), t\right) .
$$

From the definition of $U$, we have

$$
v(x, t)=\frac{1}{2 \sqrt{\pi}} \int_{0}^{t} \frac{\hat{\sigma}_{\xi}\left(x_{-}(\tau), \tau\right)}{\sqrt{t-\tau}} \mathrm{e}^{-\frac{\left(x-x_{-}(\tau)\right)^{2}}{4(t-\tau)}} \mathrm{d} \tau .
$$

Take $\alpha$ is sufficiently close to $t$, and the absolute value of integration on the curve $\Gamma_{\alpha t}$ is

$$
\begin{aligned}
\left|\frac{1}{2 \sqrt{\pi}} \int_{0}^{t} \frac{\hat{\sigma}_{\xi}\left(x_{-}(\tau), \tau\right)}{\sqrt{t-\tau}} \mathrm{e}^{-\frac{\left(x-x_{-}(\tau)\right)^{2}}{4(t-\tau)}} \mathrm{d} \tau\right| & \leq \frac{1}{2 \sqrt{\pi}} \int_{\alpha}^{t} \frac{\left|\hat{\sigma}_{\xi}\left(x_{-}(\tau), \tau\right)\right|}{\sqrt{t-\tau}} \mathrm{e}^{\frac{\left(x-x_{-}(\tau)\right)^{2}}{4(t-\tau)}} \mathrm{d} \tau \\
& \leq \frac{1}{2 \sqrt{\pi}} \int_{\alpha}^{t} \frac{\left|\hat{\sigma}_{\xi}\left(x_{-}(\tau), \tau\right)\right|}{\sqrt{t-\tau}} \mathrm{d} \tau \\
& \leq \frac{M}{2 \sqrt{\pi}} \int_{\alpha}^{t} \frac{1}{\sqrt{t-\tau}} \mathrm{d} \tau=\frac{M}{\sqrt{\pi}} \sqrt{t-\alpha} .
\end{aligned}
$$

Since, $\alpha$ is sufficiently close to $t$, and the absolute value of $v(x, t)$ is sufficiently small, hence, $v$ is uniformly converges on any point close to $x_{-}(t)$.

Now, prove the continuity of $v$ near $\Gamma_{\alpha t}$. Especially, we want to prove that $v(x, t)$ is continuous on $\left(x_{-}(t), t\right)$. We prove this using the Definition of Con- 
tinuity: $\exists \delta>0,\left|x-x_{-}(t)\right|<\delta$, if

$\left|\int_{0}^{t} U\left(x, t ; x_{-}(\tau), \tau\right) \hat{\sigma}_{\xi}\left(x_{-}(\tau), \tau\right) \mathrm{d} \tau-\int_{0}^{t} U\left(x_{-}(t), t ; x_{-}(\tau), \tau\right) \hat{\sigma}_{\xi}\left(x_{-}(\tau), \tau\right) \mathrm{d} \tau\right|<\varepsilon$

holds for all $\varepsilon>0$. If we can prove there exists $\delta$ and (58) holds, then we can prove the continuity of $v(x, t)$. Now, we divide the integrals,

$$
\begin{aligned}
& \left|\int_{0}^{t} U\left(x, t ; x_{-}(\tau), \tau\right) \hat{\sigma}_{\xi}\left(x_{-}(\tau), \tau\right) \mathrm{d} \tau-\int_{0}^{t} U\left(x_{-}(t), t ; x_{-}(\tau), \tau\right) \hat{\sigma}_{\xi}\left(x_{-}(\tau), \tau\right) \mathrm{d} \tau\right| \\
& =\mid \int_{\alpha}^{t} U\left(x, t ; x_{-}(\tau), \tau\right) \hat{\sigma}_{\xi}\left(x_{-}(\tau), \tau\right) \mathrm{d} \tau-\int_{\alpha}^{t} U\left(x_{-}(t), t ; x_{-}(\tau), \tau\right) \hat{\sigma}_{\xi}\left(x_{-}(\tau), \tau\right) \mathrm{d} \tau \\
& \int_{0}^{\alpha} U\left(x, t ; x_{-}(\tau), \tau\right) \hat{\sigma}_{\xi}\left(x_{-}(\tau), \tau\right) \mathrm{d} \tau-\int_{0}^{\alpha} U\left(x_{-}(t), t ; x_{-}(\tau), \tau\right) \hat{\sigma}_{\xi}\left(x_{-}(\tau), \tau\right) \mathrm{d} \tau \mid \\
& \leq\left|\int_{\alpha}^{t} U\left(x, t ; x_{-}(\tau), \tau\right) \hat{\sigma}_{\xi}\left(x_{-}(\tau), \tau\right) \mathrm{d} \tau\right|+\left|\int_{\alpha}^{t} U\left(x_{-}(t), t ; x_{-}(\tau), \tau\right) \hat{\sigma}_{\xi}\left(x_{-}(\tau), \tau\right) \mathrm{d} \tau\right| \\
& +\left|\int_{0}^{\alpha} U\left(x, t ; x_{-}(\tau), \tau\right) \hat{\sigma}_{\xi}\left(x_{-}(\tau), \tau\right) \mathrm{d} \tau-\int_{0}^{\alpha} U\left(x_{-}(t), t ; x_{-}(\tau), \tau\right) \hat{\sigma}_{\xi}\left(x_{-}(\tau), \tau\right) \mathrm{d} \tau\right| .
\end{aligned}
$$

where $\alpha$ is sufficiently close to $t$. ( $t-\alpha$ is sufficiently small). Therefore, from the convergence of $v(x, t)$, we can write, for very small $t-\alpha$, it holds that

$$
\begin{gathered}
\left|\int_{\alpha}^{t} U\left(x, t ; x_{-}(\tau), \tau\right) \hat{\sigma}_{\xi}\left(x_{-}(\tau), \tau\right) \mathrm{d} \tau\right|<\varepsilon_{1}, \\
\left|\int_{\alpha}^{t} U\left(x_{-}(t), t ; x_{-}(\tau), \tau\right) \hat{\sigma}_{\xi}\left(x_{-}(\tau), \tau\right) \mathrm{d} \tau\right|<\varepsilon_{1},
\end{gathered}
$$

for all $\varepsilon_{1}$. Furthermore, when $t-\alpha$ is sufficiently small, then $x-x_{-}(t)$ is sufficiently small. Next from the continuity of $U(x, t ; \xi, \tau)$,

$$
\begin{aligned}
& \left|\int_{0}^{\alpha} U\left(x, t ; x_{-}(\tau), \tau\right) \hat{\sigma}_{\xi}\left(x_{-}(\tau), \tau\right) \mathrm{d} \tau-\int_{0}^{\alpha} U\left(x_{-}(t), t ; x_{-}(\tau), \tau\right) \hat{\sigma}_{\xi}\left(x_{-}(\tau), \tau\right) \mathrm{d} \tau\right| \\
& \leq \int_{0}^{\alpha}\left|U\left(x, t ; x_{-}(\tau), \tau\right)-U\left(x_{-}(t), t ; x_{-}(\tau), \tau\right)\right|\left|\hat{\sigma}_{\xi}\left(x_{-}(\tau), \tau\right)\right| \mathrm{d} \tau \\
& \leq \hat{\varepsilon} \cdot M \cdot|\alpha-0|=\varepsilon_{2} .
\end{aligned}
$$

Therefore (58) holds, which implies

$$
\lim _{x \rightarrow x_{-}(t)+0} v(x, t)=v\left(x_{-}(t), t\right) .
$$

c) Conformation of

$$
\lim _{x_{-}(t)+0} \int_{0}^{t} U_{\xi}\left(x, t ; x_{-}(\tau), \tau\right) \hat{\sigma}\left(x_{-}(\tau), \tau\right) \mathrm{d} \tau=\int_{0}^{t} U_{\xi}\left(x_{-}(t), t ; x_{-}(\tau), \tau\right) \hat{v}_{-}(\tau) \mathrm{d} \tau+\frac{1}{2} \hat{v}_{-}(t)
$$

where, $\hat{\sigma}_{\xi}$ is bounded and lipschitz continuous.

Proof. From the definition of $U$, we have

$$
\begin{aligned}
& \int_{0}^{t} U_{\xi}\left(x, t ; x_{-}(\tau), \tau\right) \hat{\sigma}_{\xi}\left(x_{-}(\tau), \tau\right) \mathrm{d} \tau \\
& =\frac{1}{4 \sqrt{\pi}} \int_{0}^{t} \frac{x-x_{-}(\tau)}{\sqrt{(x-\tau)^{3}}} \hat{\sigma}_{\xi}\left(x_{-}(\tau), \tau\right) \mathrm{e}^{-\frac{\left(x-x_{-}(\tau)\right)^{2}}{4(t-\tau)}} \mathrm{d} \tau \\
& =\frac{1}{\sqrt{\pi}} \int_{\frac{x-x_{-}(0)}{2 \sqrt{t}}}^{+\infty} \frac{x-x_{-}(\tau)}{t-\tau}\left[\frac{1}{-2 x_{-}^{\prime}(\tau)+\frac{x-x_{-}(\tau)}{t-\tau}}\right] \hat{\sigma}_{\xi}\left(x_{-}(\tau), \tau\right) \mathrm{e}^{-\delta^{2}} \mathrm{~d} \delta,
\end{aligned}
$$


where, $\delta=\frac{x-x_{-}(\tau)}{2 \sqrt{t-\tau}}$. For simplicity, set $\hat{\sigma}_{\xi} \equiv 1$, and

$$
w_{0}(x, t)=\frac{1}{\sqrt{\pi}} \int_{\frac{x-x_{-}(0)}{2 \sqrt{t}}}^{+\infty} \frac{x-x_{-}(\tau)}{t-\tau}\left[\frac{1}{-2 x_{-}^{\prime}(\tau)+\frac{x-x_{-}(\tau)}{t-\tau}}\right] \mathrm{e}^{-\delta^{2}} \mathrm{~d} \delta .
$$

Suppose,

$$
w_{1}(x, t)=\frac{1}{\sqrt{\pi}} \int_{\frac{x-x_{-}(0)}{2 \sqrt{t}}}^{+\infty}\left[\frac{1}{-2 x_{-}^{\prime}(\tau)+\frac{x-x_{-}(\tau)}{t-\tau}}\right] \mathrm{e}^{-\delta^{2}} \mathrm{~d} \delta
$$

hence,

$$
w_{0}(x, t)+w_{1}(x, t)=\frac{1}{\sqrt{\pi}} \int_{\frac{x-x_{-}(0)}{2 \sqrt{t}}}^{++\infty} \mathrm{e}^{-\delta^{2}} \mathrm{~d} \delta,
$$

when $x \rightarrow x_{-}(t)+0$, we have

$$
\lim _{x \rightarrow x_{-}(t)+0} w_{0}(x, t)+\lim _{x \rightarrow x_{-}(t)+0} w_{1}(x, t)=\frac{1}{\sqrt{\pi}} \int_{\frac{x_{-}(t)-x_{-}(0)}{2 \sqrt{t}}}^{+\infty} \mathrm{e}^{-\delta^{2}} \mathrm{~d} \delta .
$$

When $x=x_{-}(t)$ and $\tau=t$, we have

$$
w_{0}\left(x_{-}(t), t\right)+w_{1}\left(x_{-}(t), t\right)=\frac{1}{\sqrt{\pi}} \int_{\frac{x_{-}(t)-x_{-}(0)}{2 \sqrt{t}}}^{0} \mathrm{e}^{-\delta^{2}} \mathrm{~d} \delta,
$$

next, from (59) and (60), we can get

$$
\begin{aligned}
& \lim _{x \rightarrow x_{-}(t)+0} w_{0}(x, t) \\
& =w_{0}\left(x_{-}(t), t\right)-\left[\lim _{x \rightarrow x_{-}(t)+0} w_{1}(x, t)-w_{1}\left(x_{-}(t), t\right)\right]+\frac{1}{\sqrt{\pi}} \int_{0}^{+\infty} \mathrm{e}^{-\delta^{2}} \mathrm{~d} \delta \\
& =w_{0}\left(x_{-}(t), t\right)+\frac{1}{2},
\end{aligned}
$$

since,

$w_{1}(x, t)=\frac{1}{\sqrt{\pi}} \int_{\frac{x-x_{-}(0)}{2 \sqrt{t}}}^{+\infty}\left[\frac{1}{-2 x_{-}^{\prime}(\tau)+\frac{x-x_{-}(\tau)}{t-\tau}}\right] \mathrm{e}^{-\delta^{2}} \mathrm{~d} \delta=\frac{1}{4 \sqrt{\pi}} \int_{0}^{t} \frac{-2 x_{-}^{\prime}(\tau)}{\sqrt{t-\tau}} \mathrm{e}^{-\frac{\left(x-x_{-}(\tau)\right)^{2}}{4(t-\tau)}} \mathrm{d} \tau$.

From the proof of Appendix B, one can know $w_{1}(x, t)$ is continuous near $x_{-}(t)$, therefore,

$$
\lim _{x \rightarrow x_{-}(t)+0} w_{1}(x, t)=w_{1}\left(x_{-}(t), t\right) .
$$

Finally, we get

$$
\begin{aligned}
& \lim _{x \rightarrow x_{-}(t)+0} \frac{1}{4 \sqrt{\pi}} \int_{0}^{t} \frac{x-x_{-}(\tau)}{\sqrt{(t-\tau)^{3}}} \mathrm{e}^{-\frac{\left(x-x_{-}(\tau)\right)^{2}}{4(t-\tau)}} \mathrm{d} \tau \\
& =\frac{1}{4 \sqrt{\pi}} \int_{0}^{t} \frac{x_{-}(t)-x_{-}(\tau)}{\sqrt{(t-\tau)^{3}}} \mathrm{e}^{-\frac{\left(x_{-}(t)-x_{-}(\tau)\right)^{2}}{4(t-\tau)}} \mathrm{d} \tau+\frac{1}{2},
\end{aligned}
$$


where, $\hat{\sigma}_{\xi}\left(x_{-}(\tau), \tau\right) \equiv 1$. From Appendix B, we have

$$
\begin{aligned}
& \lim _{x \rightarrow x_{-}(t)+0} \int_{0}^{t} U_{\xi}\left(x, t ; x_{-}(\tau), \tau\right) \hat{\sigma}_{\xi}\left(x_{-}(\tau), \tau\right) \mathrm{d} \tau \\
= & \int_{0}^{t} U_{\xi}\left(x_{-}(t), t ; x_{-}(\tau), \tau\right) \hat{v}_{-}(\tau) \mathrm{d} \tau+\frac{1}{2} \hat{v}_{-}(t),
\end{aligned}
$$

Next, we can get

$$
U_{\xi}\left(x, t ;-x_{-}(\tau), \tau\right) \hat{\sigma}_{\xi}\left(x_{-}(\tau), \tau\right) \mathrm{d} \tau
$$

since, $-x_{-}(\tau)$ is away from $x_{-}(t)$ and the integral

$$
\int_{0}^{t} U_{\xi}\left(x, t ;-x_{-}(\tau), \tau\right) \hat{\sigma}_{\xi}\left(x_{-}(\tau), \tau\right) \mathrm{d} \tau,
$$

is continuous on $x_{-}(t)$.

Submit or recommend next manuscript to SCIRP and we will provide best service for you:

Accepting pre-submission inquiries through Email, Facebook, LinkedIn, Twitter, etc. A wide selection of journals (inclusive of 9 subjects, more than 200 journals)

Providing 24-hour high-quality service

User-friendly online submission system

Fair and swift peer-review system

Efficient typesetting and proofreading procedure

Display of the result of downloads and visits, as well as the number of cited articles Maximum dissemination of your research work

Submit your manuscript at: http://papersubmission.scirp.org/

Or contact ojapps@scirp.org 